\title{
Immune age and biological age as determinants of vaccine responsiveness among elderly populations: the Human Immunomics Initiative research program
}

\author{
Jaap Goudsmit ${ }^{1,2,3} \cdot$ Anita Huiberdina Johanna van den Biggelaar ${ }^{3}\left(\mathbb{D} \cdot\right.$ Wouter Koudstaal $^{3} \oplus$ - Albert Hofman ${ }^{1}(\mathbb{D}$.

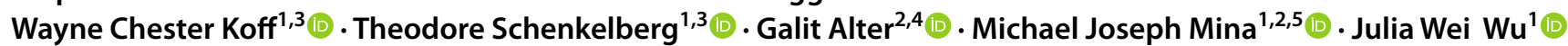

Received: 17 November 2020 / Accepted: 27 May 2021 / Published online: 12 June 2021

(c) The Author(s) 2021

\begin{abstract}
The Human Immunomics Initiative (HII), a joint project between the Harvard T.H. Chan School of Public Health and the Human Vaccines Project (HVP), focuses on studying immunity and the predictability of immuneresponsiveness to vaccines in aging populations. This paper describes the hypotheses and methodological approaches of this new collaborative initiative. Central to our thinking is the idea that predictors of age-related non-communicable diseases are the same as predictors for infectious diseases like COVID-19 and influenza. Fundamental to our approach is to differentiate between chronological, biological and immune age, and to use existing large-scale population cohorts. The latter provide well-typed phenotypic data on individuals' health status over time, readouts of routine clinical biochemical biomarkers to determine biological age, and bio-banked plasma samples to deep phenotype humoral immune responses as biomarkers of immune age. The first phase of the program involves 1 . the exploration of biological age, humoral biomarkers of immune age, and genetics in a large multigenerational cohort, and 2. the subsequent development of models of immunity in relation to health status in a second, prospective cohort of an aging population. In the second phase, vaccine responses and efficacy of licensed COVID-19 vaccines in the presence and absence of influenza-, pneumococcal- and pertussis vaccines routinely offered to elderly, will be studied in older aged participants of prospective population-based cohorts in different geographical locations who will be selected for representing distinct biological and immune ages. The HII research program is aimed at relating vaccine responsiveness to biological and immune age, and identifying aging-related pathways crucial to enhance vaccine effectiveness in aging populations.
\end{abstract}

Keywords Immune aging $\cdot$ Biological age $\cdot$ Aging-related diseases $\cdot$ Vaccine responsiveness

Anita Huiberdina Johanna van den Biggelaar

anita.vandenbiggelaar@gmail.com

1 Department of Epidemiology, Harvard T.H. Chan School of Public Health, Boston, MA, USA

2 Department of Immunology and Infectious Diseases, Harvard T.H. Chan School of Public Health, Boston, MA, USA

3 Human Vaccines Project, New York, NY, USA

4 Ragon Institute of MGH, MIT and Harvard, Cambridge, MA, USA

5 Department of Pathology, Brigham and Women's Hospital, Harvard Medical School, Boston, USA

\section{Introduction}

Infectious diseases present a major threat to the health of elderly populations. Compared to younger populations, older adults are at greater risk of developing severe symptoms, requiring hospitalization, and dying from infections. This is most evident in the current COVID-19 pandemic, where age is a strong predictor of the severity and outcome of SARS$\mathrm{CoV}-2$ infections, with adults aged over 65 years representing $80 \%$ of COVID-19 hospitalizations and having a more than 20-fold greater risk to die of COVID-19 [1,2]. But also for other respiratory infections, such as influenza [3, 4], pneumococcal pneumonia $[3,5]$, and pertussis $[6,7]$, and other viral and bacterial infections including bacteraemia $[8,9]$ and severe gastroenteritis, risks exponentially increase above the age of $65[10,11]$. 
Main factors contributing to the increased vulnerability at older age include higher exposure, aging of the immune system, and co-morbidities [12-15]. Higher exposure primarily occurs through increased utilization of healthcare and long-term care facilities, where the risk of transmission and infection are high $[16,17]$. Also the risk of re-activation of dormant infections such as herpes zoster [18] and tuberculosis [19] increases with age. The latter clearly signifies a failure of the aging immune system to control infections as a consequence of immunosenescence, i.e. the gradual deterioration of the immune system with natural age advancement. Another consequence of immune aging is a reduced ability to elicit effective immune responses to vaccines, as has been demonstrated for pneumococcal polysaccharide vaccines [20,21], seasonal influenza vaccine [8], and herpes zoster vaccine [22]. Reduced vaccine responsiveness further limit the potential to protect older adults against severe infectious diseases. Finally, older adults developing severe infections often suffer from one or more non-communicable diseases, such as hypertension, ischemic heart disease, stroke, diabetes mellitus, chronic kidney disease, autoimmune, and neurodegenerative diseases: conditions that all

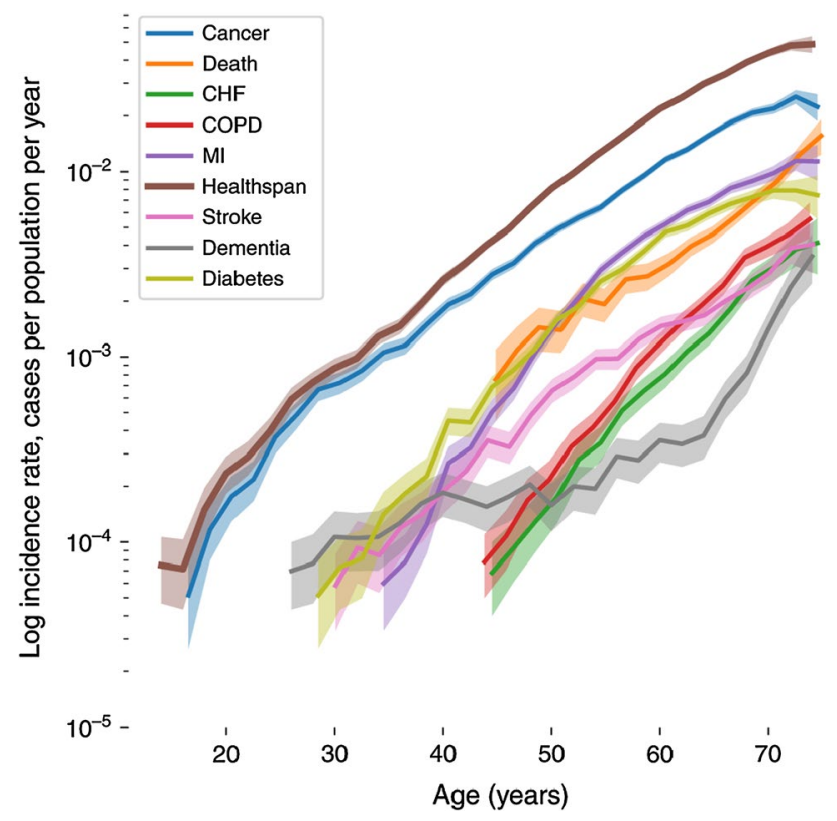

Fig. 1 Incidence rates of the most prevalent chronic diseases, death, and healthspan based on clinical histories for over 300,000 people, aged 37 to 73 years old, participating in the UK Biobank cohort. Incidence rates for different chronic diseases, healthspan, and death increase at comparable, approximately exponentially rates with age. Disease incidence rates were calculated independently, with participants who develop more than one condition during the follow-up period counting for every disease they have. Healthspan was defined based in the first illness event occurrings. Shaded areas represent $95 \%$ confidence intervals. The graph was reproduced from Zenin et al., Identification of 12 genetic loci associated with human healthspan. Commun Biol. 2019 Jan 30;2:41 increase exponentially at older age, as shown in Fig. 1 for UK Biobank participants [23].

The risks of developing non-communicable and communicable diseases with increasing age are not independent of each other: as clearly shown for COVID-19, pre-existing illnesses such as diabetes and hypertension are independent risk factors for developing and dying of a severe infection [24]. Both age-related non-communicable diseases and severe infectious diseases are associated with a chronic state of low-grade inflammation [25], which presents an important feature of the aging immune system known as 'inflammaging'.

The 'immunome' is defined as the detailed map of immune reactions of a given host interacting with a foreign antigen, and 'immunomics' as the study of immunomes [26, 27]. In this paper we describe the objectives, hypotheses and methodological approaches of The Human Immunomics Initiative (HII), a joint project between the Harvard T.H. Chan School of Public Health and the Human Vaccines Project (HVP). The aim of HII is to decode the mechanisms and rules of effective immunity in aging populations through the exploration in large-scale population cohorts of biomarkers of immunosenescence, inflammaging, and risks for infectious and non-infectious diseases, and ultimately vaccine responses, in relationship to age. The differentiation between chronological and biological age is key in this process. Given the role of the immune system in age-related non-communicable diseases, HII takes the view that immunomics should not be limited to immune reactions with foreign antigens, but include also immune reactions to (modified) self antigens.

\section{Biological age as a predictor of all-cause mortality and age-related non-communicable diseases}

Aging is characterized by a progressive loss of intrinsic capacity and functional ability, and increased risk for morbidities and death. Chronological age is an important predictor of morbidity and mortality but cannot account for heterogeneity in the decline of physiological function and health with advancing age. The realization that the rate at which people age is not universal, led to the concept that people have a biological age that reflects an individual's global physiological status and functioning, his/her susceptibility to death and disease, and as such is a better predictor of lifespan and health span than chronological age [28-32].

In recent years, many studies have invested in studying biomarkers defining and predicting biological age, or hallmarks of aging [28, 33, 34]. Many of these studies have focused on biomarkers presenting a measure of biological age that is predictive of all-cause mortality. This 
includes physiological and biochemical biomarkers, such as for example the study of Levine et al. that used ten biomarkers: i.e. C-reactive protein, serum creatinine, glycated hemoglobin, systolic blood pressure, serum albumin, total cholesterol, cytomegalovirus optical density, serum alkaline phosphatase, forced expiratory volume, and serum urea nitrogen [28]. Other examples of biomarkers are systolic blood pressure, pulmonary vital capacity, creatinine, fasting glucose, as well as a Modified MiniMental Status Examination score presenting a 'Healthy Aging Score'[33], molecular or epigenetic markers such as telomere length [35] and DNA methylation [29], or metabolomic predictors [36]. Biological age should, however, also account for differences in the physiological status and risk for age-related diseases among individuals of the same chronological age. More importantly, measures of biological aging based on clinically observable data, as opposed to those using molecular measures such as epigenetic clocks and leukocyte telomere length, tend to better capture risks for death and diseases and to be more robust predictors of aging-related outcomes [29]. By studying clinical measures representative of the physiological status of multiple organ systems (e.g. pulmonary, periodontal, cardiovascular, renal, hepatic, and immune function) repeatedly over a period of 12 years in middleaged adults (the Dunedin Study), Belsky et al. [30] showed that already at midlife, before the onset of age-related diseases, an individual's chronological age and biological age are divergent measures, with those who age more rapidly (i.e. who have an older biological age) being physically less able, showing cognitive decline and brain aging, selfreporting worse health, and looking older (Fig. 2).

Levine et al. [29] showed that biological age can differentiate between morbidity and mortality risks among chronological same-aged individuals. They further showed that using nine multi-system clinical chemistry biomarkers (albumin, creatinine, glucose, C-reactive protein, lymphocyte percent, mean cell volume, red blood cell distribution width, alkaline phosphatase, and white blood cell count), the difference between an individual's biological versus chronological age is highly predictive of mortality, although the size of this effect decreases with age [37]. Wu et al. recently showed that physiological composite scorebased biological age and its deviation from chronological age capture risks of death and all major aging-related morbidity, including dementia (manuscript submitted). Basic to the definition of biological age in the HII program is the risk-capturing summary of routine multi-system clinical chemistry biomarkers as initially introduced by Levine that are generally available for large scale population cohorts. Because of its robustness in predicting aging-related outcomes, we consider Wu's physiological composite

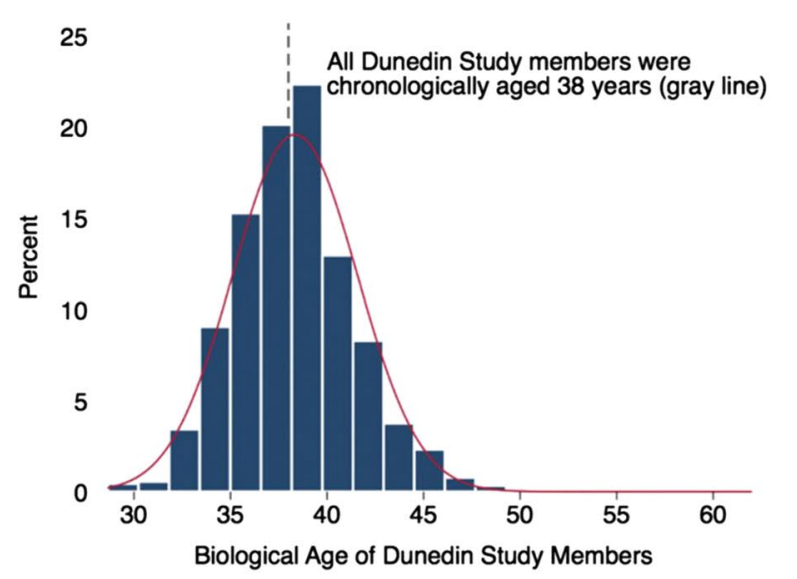

Fig. 2 Biological versus chronological age in the Dunedin Study including 1037 young adults followed from birth to age 38 years. Biological age is normally distributed in a cohort of adults aged 38 years (left). Healthy adults who were aging faster exhibited deficits in physical functioning, showed evidence of cognitive decline, felt less healthy and were rated as looking older by independent observers (right). The figure shows binned scatter plots of the associations of
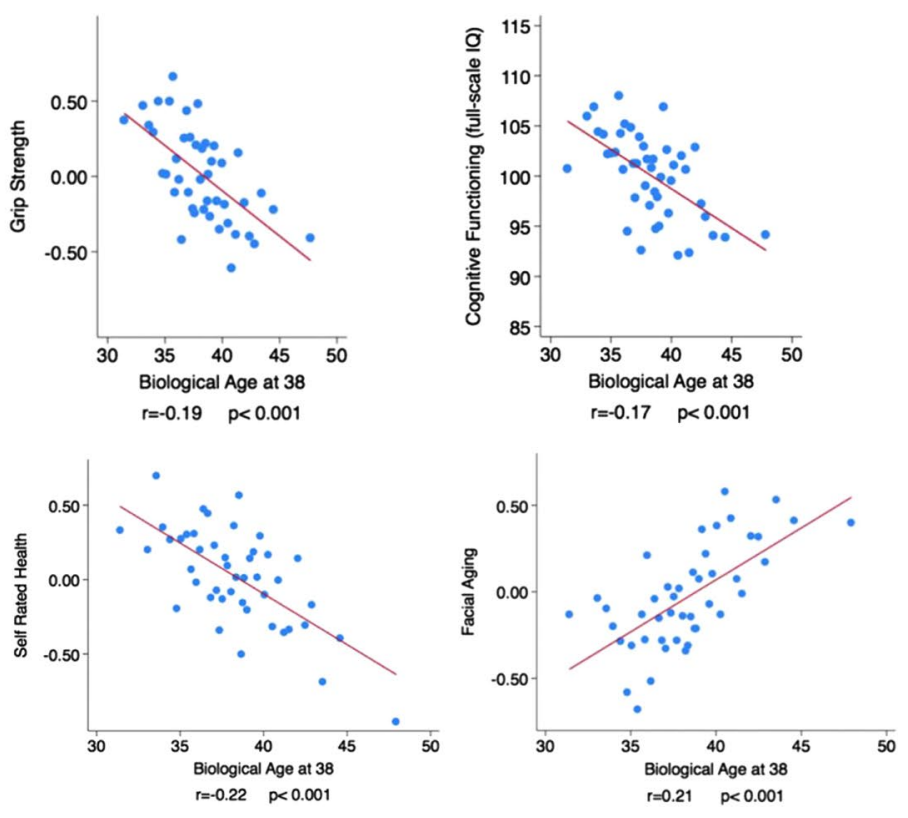

biological age with grip strength, cognitive functioning, self-rated health and with facial aging. Each plotted dot point shows the mean for bins of data from $N=20$ Dunedin Study members. Effect size and regression line were calculated from the raw data. Adapted with permission from Belsky WD et al., Quantification of biological aging in young adults. Proc Natl Acad Sci USA. 2015 Jul 28;112(30):E410410 
score-based biological age and its deviation from chronological age a better calibration tool for studying mechanistic aging than chronological age.

\section{Biological age as predictor of infectious diseases}

As mentioned before, biological aging has been shown to be a stronger predictor for all-cause mortality and mortality from non-communicable diseases than chronological aging. However, it was not until the current COVID-19 pandemic that associations between biological aging and the risk of mortality from infectious diseases were studied. Since COVID-19 emerged, it has become clear that people of older age, and in particular those with co-morbidities, are at higher risk to develop and die of severe COVID$19[1,2,24]$. Applying the previously validated measure of biological age that was based on nine routine clinical biochemical markers, Levine and colleagues demonstrated that faster biological aging is associated with COVID-19 severity [38]. Importantly, Levine et al. did not determine biological age in people at the time they were diagnosed with COVID-19, but assessed their biological age 10-to14 years prior to the onset of COVID-19. This was possible because the COVID-19 patients were previous participants of a large community cohort including over 500,000 subjects recruited between the ages of 40 and 70 during 2006 to 2010 in the United Kingdom (UK Biobank), and had routine clinical biomarkers determined at the time of recruitment. The observation that the deviation of biological age from chronological age is a strong predictor for developing a severe infectious illness a decade or more later, is novel and supports the notion that accelerated biological aging is a strong predictor of severe disease at older age, including infectious diseases.

\section{Immune age}

Ahadi et al. [39] described four 'ageotypes', or biological pathways of aging, including: immunity, metabolic pathways, liver dysregulation, and kidney dysregulation. For each of these pathways, people may age at different rates. Like for other systems, changes in immune functioning over lifetime are likely less dictated by chronological age than by individual trajectories that may be influenced by genetics, epigenetics, and environmental factors [40-42]. 'Immune age' may therefore be a predictor of infectious and non-communicable diseases and vaccine responsiveness at older age.
The human immune system involves more than 1500 genes/proteins in many interconnected pathways and processes [43]. The last decade has seen an explosion in highthroughput technologies that allow us to study the human immune system in small volumes of blood based on the detection of multi-level changes in molecular immune pathways and networks. This includes but is not limited to platforms for RNA-sequencing, flowcytometry, and highperformance liquid chromatography or mass spectometry for plasma proteomics and antibody glycosylation [44, 45]. Consequently, a holistic study of the immune system in relation to individual and population health and disease is now technically possible, and with that the opportunity to phenotype Immune Age. Pulendran and colleagues [22] used this approach to explore immune pathways in response to Herpes Zoster vaccination in both younger and older adults, and identified immune and metabolic correlates predictive of differences in vaccine immunity in older vs younger adults [22]. Another example is a study by Alpert et al. [46] who used an 'omics' approach applied to a longitudinal cohort of adults sampled multiple times over the course of nine years, to identify an immune age algorithm that was predictive of all-cause mortality. Pulendran and Alpert both used isolated immune cell populations to phenotype cell gene expression profiles (transcriptomics). Access to isolated blood immune cells, however, requires specifically designed, smaller-scale studies. Usually immune cells are not available from large communitybased population cohorts. In contrast, plasma samples are typically bio-banked at larger quantities. High-throughput platforms to analyse functional properties of antibody responses are now available and have been used to study immunity in vaccinated or infected humans [47, 48].

Antibodies play an important role in both immunosenescence and inflammaging: two processes that both define immune aging [49]. As mentioned, immunosenescence is an age-related weakening of the immune system's ability to respond to danger signals and clear pathogens. One aspect of immunosenescence is a narrowing of the B-cell repertoire against non-self-antigens, and a failure of self-tolerance mechanisms to deplete B-cells recognizing self-antigens. As a result, the spectrum of antibodies recognizing pathogens and danger signals becomes smaller, while the spectrum of auto-antibodies is thought to increase with aging [50,51]. Inflammaging is an age-related development of a chronic state of low-grade inflammation that is caused by a sustained activation of innate immune cells. Inflammasomes may play an important role in inflammaging and age-related diseases $[52,53]$.

Inflammasomes are protein complexes that are formed when specific receptors of innate immune cells recognize microbial or danger signals. Inflammasomes were first discovered by the team of Tschopp et al. [54] and a more 
detailed description on inflammasomes and their function can be found in a review by Schroder and Tschopp. In brief, inflammasomes stimulate the production of the pro-inflammatory cytokines IL-1ß and IL-18, and lead to a pro-inflammatory type of cell death known as pyroptosis. The assumed primary role of inflammasomes is to mediate protection against invading pathogens, with pyroptosis leading to the death of infected cells and activation of adaptive immune responses to clear infection. A number of host mechanisms also suppress inflammasome activation, presumably in order to limit the extent of potentially dangerous immune activation. Aberrations in inflammasome-mediated signaling and control mechanisms may result in increased susceptibility for infections and/or more severe disease symptoms, and could predispose to developing autoreactive immune responses. This may involve antibodies that can potentiate or suppress the inflammasome $[47,55,56]$.

Antibody functionality is in part regulated through glycan structures present on the antibody's constant region. Hundreds of differentially glycosylated antibody variants can be present in an individual at any given time, some of which are associated with pro-inflammatory responses and others with anti-inflammatory properties. This large individual variability contributes to individual differences in function of the immune system. Aging, auto-immunity, non-communicable, and severe infectious diseases are all associated with immunoglobulin $\mathrm{G}(\mathrm{IgG})$ glycan species promoting inflammatory responses [47, 49, 57-59]. Glycans therefore may regulate the potentiating or suppressing effect of antibodies on innate immune responses. At the same time, antibody glycans may contribute to immune senescence as glycan species expressed at older age may be associated with lower receptor binding and affinity, and hence possibly reduced pathogen clearance $[58,60]$.

By analysing $\operatorname{IgG}$ glycosylation in relation to chronological age in a training set and in a subsequent validation set including more than 5000 individuals from four different European populations, Krištić et al. [61] developed a predictive model consisting of only three glycans, called 'Glycan Age': this model can predict chronological age with an error of 9.7 years, and explain nearly $60 \%$ of variation in chronological age and sex. In comparison, conservative age biomarkers such as telomere length accounted for as little as $15 \%$ to $25 \%$ of variance of age in this study. The "Glycan Age" index was also found to correlate with physiological markers that change with aging and possible predictors of biological age. This suggest that antibody glycosylation may be a predictor of biological age, and potent biomarker of immune age: two hypotheses that are fundamental and will be studied in the HII program.

Antibody glycosylation profiles that are progressively seen with aging are also associated with metabolic health [62]. Metabolic processes play both direct and indirect roles in inflammaging and age-related infectious and non-infectious diseases [36, 63, 64]. Immune aging, risk for severe infectious diseases, and metabolomic health therefore are likely interrelated, and may be mediated through similar changes in antibody glycosylation. This interconnectivity of immune aging and metabolic pathways has become evident in recent studies of severe COVID-19 outcomes in older patients [25, 65-70], where inflammasome activation [71] and altered antibody glycosylation [72, 73] have been associated with a reduced capacity of older individuals to clear the SARS-CoV-2 virus [74] and subsequent enhancement of hyper-inflammation responses [75]. Emerging observations that COVID-19 has the potential to enhance and accelerate processes of immunosenescence and inflammaging [76] is novel evidence that infectious diseases can negatively impact immune age. Hence, immune age may depend to a certain extent on infections that individuals have experienced in the past. Immunity and the ability to respond effectively to vaccination is thus shaped by a complex of interacting factors, including aging, immunosenescence, inflammaging, metabolomics and antibody glycosylation.

\section{The HII program}

In order to decode mechanisms and rules of effective immunity in aging populations, the Human Immunomics Initiative (HII) will leverage the strength of large prospective population cohorts that provide a wealth of information on the health status and clinical endpoints of a large number of participants who are representative for the general population. Given that bio-banks of population cohorts typically store plasma but not immune cells, HII will focus on highthroughput analyses that can be conducted using plasma to identify biomarkers of immune age and phenotype humoral immuneresponses. This variant of "systems serology" [77, 78] may include: phage-immunoprecipitation-sequencing (PhIP-Seq)-based methods for the comprehensive analysis of serum antibodies to human pathogens ("VirScan") and self antigens ("peptidome") developed by Stephen Elledge [79-83]; antibody glycosylation analysis as described above for Glycan age and in a further expanded approach, and additional analyses such as transcriptomics and metabolomics. Next to system serology, biological age as determined from routine clinical biochemical parameters will be used to characterize the background of the aging person. When available, genetics will also be considered, as genetic loci have been shown to be associated with healthspan [23] as well as with glycosylation of IgG [84], and thus immune age (Fig. 3).

The program will consist of two phases, with phase 1 aimed at exploring and validating the concept of immune age as based on systems serology parameters in relation to 


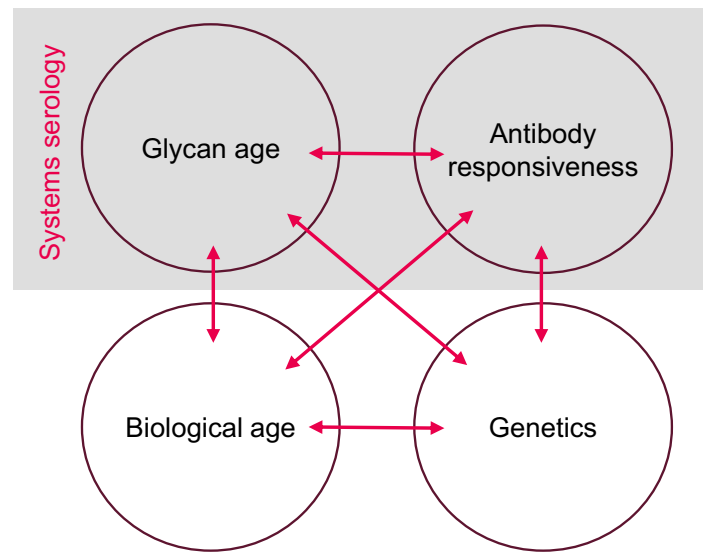

Fig. 3 Set of interacting factors shaping the aging immune system studied by HII

biological age and age-related illnesses. In the second phase, biological age and immune age will be studied as predictors of vaccine responsiveness in vaccine studies nested within large prospective population-based cohorts in different geographical locations worldwide.

The first step of phase 1 will explore the association of chronological age, genetics, biological age and immune age based on glycan age and antibody responsiveness in a large-scale prospective cohort study. The model of biological age as developed by Levine, and later further built upon by Wu using routine clinical biomarkers, will be applied to select from each age group (e.g. participants aged $15 \pm 2$ years; $25 \pm 2$ years; etc. up to $85 \pm 2$ years at the time of sampling) subjects who are at the extremes of biological age, i.e. who have the youngest or oldest biological age for their chronological age. Plasma samples from these subjects will then be analysed to define immune age based on antibody glycosylation analysis and deep serological profiling for non-self and self-antibodies (Fig. 4). This first phase is planned to be conducted in 2021 .

In the second step of phase 1, the HII program aims to study the immune status at advanced age as characterized by biological age, glycan age and antibody responsiveness, as predictor of healthspan and life span in community-based cohorts of aged adults. Plasma samples will be analysed for immune age using the same technologies as in the first step in the program. Modelling of these B-cell immune biomarkers, together with biomarkers of biological age and genetic markers, against mortality and morbidity risk is expected to provide an optimised measure of immune age that is predictive of healthspan and lifespan (Fig. 5).

In phase 2 of the HII program we will focus on translating the learnings of phase 1 on effective immunity at older age to a current real-life situation of protecting vulnerable aging population against infectious diseases, including COVID19. Like phase 1, phase 2 of the program will be conducted nested within existing population cohorts. The same instruments and analytical assays as used and validated in phase 1 will be applied to determine biological age and immune age. The aim is not to conduct clinical vaccine trials. Instead, responses to for example COVID-19 vaccines will be studied when study subjects receive such a vaccine as part of national vaccination programs. This can also involve influenza-, pneumococcal- and pertussis vaccines that in many high-income countries are part of routine vaccination programs for elderly. The objective is to conduct this part of the program in different parts of the world, partnering with population cohorts in different geographical locations worldwide, including Europe, the US, and Asia. This means that
Selection of subjects from multigenerational cohort

- Define 8 distinct age birth cohorts

- From subjects of each discrete chronological age select 50 individuals from each of the extremes of the biological age range $(n=800)$

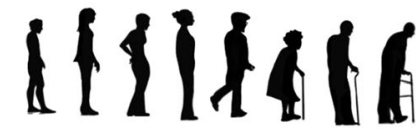

$\begin{array}{llllllll}15 & 25 & 35 & 45 & 55 & 65 & 75 & 85\end{array}$

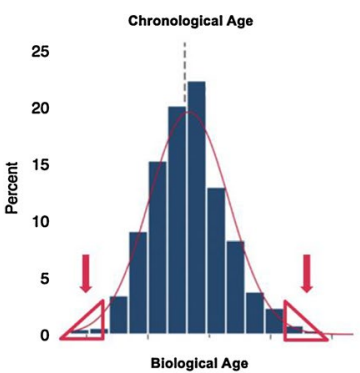

Analyses

Biological Age

Genetics

System Serology

- IgG glycosylation

- Antibodies to pathogens

- Antibodies to self-antigens

- Optional:

- Proteomics

- Metabolomics

- DNAm

- miRNA
Outcome

Artificial Intelligence and Machine Learning approaches to study associations between chronological age, biological age, glycan age and antibody responsiveness

- Proof-of-concept of technical feasibility and exploration of Immune Age algorithm

Fig. 4 Design of a phase 1 technological feasibility study to explore the associations between chronological age, biological age and immune age as defined by glycan age and antibody responsiveness and genetics 
Fig. 5 Design of phase 1 study to derive and validate an optimized measure of immune age that is predictive of healthspan and lifespan

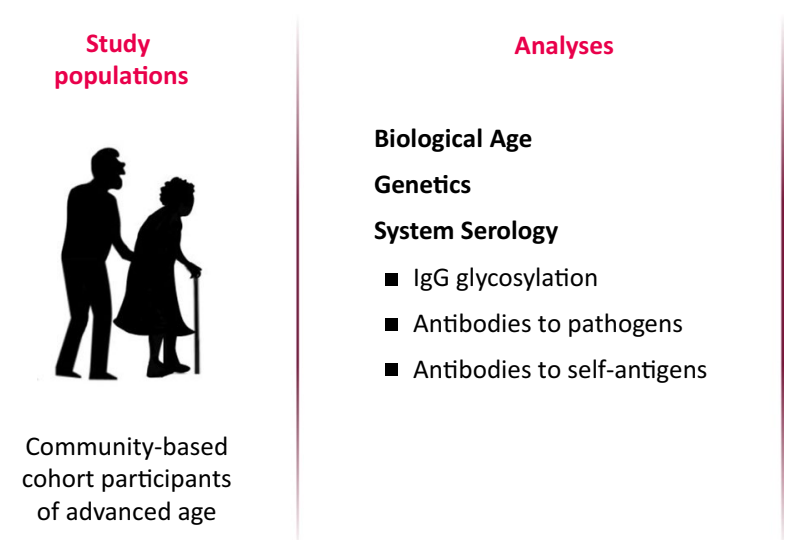

Outcome

Artificial Intelligence and Machine Learning approaches to study associations between biomarkers and communicable and noncommunicable diseases and between biomarkers and mortality

- Optimized measure of Immune Age, predictive of healthspan and lifespan
Selection of subjects from population-based cohorts

- Determine "Immune age" using optimized algorithm

- Select set of subjects of discrete advanced chronological age but divergent "immune age"

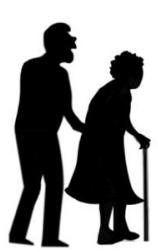

Probes

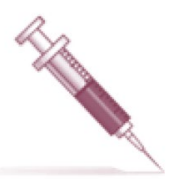

Elderly vaccines

- COVID-19

- Pneumococcal

- Influenza

- Pertussis
Outcome

Artificial Intelligence and Machine Learning approaches to study associations between biological$\&$ immune age and vaccine responsiveness

- Ability to predict vaccine responsiveness

- Identification of aging-related pathways crucial for effective immunity

Fig. 6 Design of phase 2 study to assess the predictive value of biological- and immune age algorithms for vaccine responsiveness and identification of pathways crucial for effective immunity in aging populations

we will not only study different populations but also different vaccines, as different vaccines are available in different parts of the world. From each cohort, 100-200 elderly of distinct biological and immune age will be selected. Tools that will be used to define biological and immune age will be as they are informed and optimized during Phase 1 of the program (Fig. 6).

In our proposed approach, we aim to first construct immune age composite scores based on all-cause mortality, and then evaluate their associations with risks of both non-communicable and communicable diseases including immune responsiveness to various vaccines. The resulting data will provide information regarding how consistent these associations will be. To study immunity and predictability of immune responsiveness to vaccines, immune parameters need to be considered as described in Sect. 4 . As mentioned, our "Immune Age" algorithm will be based on a selection of biomarkers from the entirety of parameters measured in the described Systems Serology that can be assessed in samples available in large-scale population cohorts (to be able to derive and validate predictive algorithms). Standard regression models are limited in their prediction capacity, given the challenge to account for complex interactions and correlation involved with high-dimensional datasets (i.e., data collections with a multitude of variables). We therefore propose to apply artificial intelligence techniques, in particular cohortbased machine learning methods [85, 86], such as elastic net regression [87], random forest models [88], and recurrent neural networks [89], for their ability to account for the complex correlation structures between multi- system serological measures. The predictive accuracy of algorithms assumes rather complete input. Another important assumption is the sufficient sample size relative to the number of parameters assessed, to avoid data overfitting. $\mathrm{K}$-fold cross-validation will be essential [85], to enhance the validation of robustness by using successive and mutually exclusive validation datasets.

The ultimate goal is to relate vaccine responsiveness as assessed by the clinical assays relevant for each of the different vaccines to biological and immune age, and identify crucial aging-related pathways that lead to 
underperformance and can potentially be adjusted, through interventions or vaccine optimization, to enhance vaccine effectiveness.

\section{Summary}

In summary, by standardization of methods and techniques to profile humoral immune responses in well-characterized human cohorts, the Human Immunomics Initiative strives to identify pathways that are crucial to improve vaccineinduced protection in aging populations. The inclusion of geographically diverse populations, which will capture variation in a population's immune status due to genetic and environmental differences, will strengthen the ability of the program to interrogate the aging human immune system and identify universal pathways of effective immunity. It is anticipated that knowledge acquired through this program can be translated directly to study outcomes for other vaccines against other serious infectious diseases that negative impact the health- and lifespan of elderly.

Authors' contributions The study concept and design was conceived by Jaap Goudsmit and developed with contribution of all authors. The first draft of the manuscript was written by Anita H. J. van den Biggelaar and all authors read and approved the final manuscript.

Funding No funding was received.

\section{Declaration}

Conflicts of interest The authors declare to have no conflicts of interest.

Open Access This article is licensed under a Creative Commons Attribution 4.0 International License, which permits use, sharing, adaptation, distribution and reproduction in any medium or format, as long as you give appropriate credit to the original author(s) and the source, provide a link to the Creative Commons licence, and indicate if changes were made. The images or other third party material in this article are included in the article's Creative Commons licence, unless indicated otherwise in a credit line to the material. If material is not included in the article's Creative Commons licence and your intended use is not permitted by statutory regulation or exceeds the permitted use, you will need to obtain permission directly from the copyright holder. To view a copy of this licence, visit http://creativecommons.org/licenses/by/4.0/.

\section{References}

1. Mueller AL, McNamara MS, Sinclair DA. Why does COVID19 disproportionately affect older people? Aging (Albany N. Y.). 2020;12(10):9959-81. doi:https://doi.org/10.18632/aging.103344

2. Santesmasses D, Castro JP, Zenin AA, et al. COVID-19 is an emergent disease of aging. Aging Cell. 2020;19(10):e13230. https://doi.org/10.1111/acel.13230.
3. Jain S, Self WH, Wunderink RG, et al. Community-acquired pneumonia requiring hospitalization among U.S. adults. N Engl J Med. 2015;373(5):415-27. https://doi.org/10.1056/NEJMoa1500245.

4. Van Kerkhove MD, Vandemaele KA, Shinde V, et al. Risk factors for severe outcomes following 2009 influenza A (H1N1) infection: a global pooled analysis. PLoS Med. 2011;8(7):e1001053. https:// doi.org/10.1371/journal.pmed.1001053.

5. Dirmesropian S, Liu B, Wood JG, et al. Pneumonia hospitalisation and case-fatality rates in older Australians with and without risk factors for pneumococcal disease: implications for vaccine policy. Epidemiol Infect. 2019;147:e118. https://doi.org/10.1017/s0950 268818003473.

6. De Serres G, Shadmani R, Duval B, et al. Morbidity of pertussis in adolescents and adults. J Infect Dis. 2000;182(1):174-9. https:// doi.org/10.1086/315648.

7. Karki S, McIntyre P, Newall AT, MacIntyre CR, Banks E, Liu B. Risk factors for pertussis hospitalizations in Australians aged 45 years and over: A population based nested case-control study. Vaccine. 2015;33(42):5647-53. https://doi.org/10.1016/j.vacci ne.2015.08.068.

8. Sasaki S, Sullivan M, Narvaez CF, et al. Limited efficacy of inactivated influenza vaccine in elderly individuals is associated with decreased production of vaccine-specific antibodies. J Clin Invest. 2011;121(8):3109-19. https://doi.org/10.1172/jci57834.

9. Laupland KB, Pasquill K, Dagasso G, Parfitt EC, Steele L, Schonheyder HC. Population-based risk factors for community-onset bloodstream infections. Eur J Clin Microbiol Infect Dis. 2020;39(4):753-8. https://doi.org/10.1007/ s10096-019-03777-8.

10. Lopman BA, Hall AJ, Curns AT, Parashar UD. Increasing rates of gastroenteritis hospital discharges in US adults and the contribution of norovirus, 1996-2007. Clin Infect Dis. 2011;52(4):466-74. https://doi.org/10.1093/cid/ciq163.

11. Verstraeten T, Cattaert T, Harris J, Lopman B, Tam CC, Ferreira G. Estimating the burden of medically attended norovirus gastroenteritis: modeling linked primary care and hospitalization datasets. J Infect Dis. 2017;216(8):957-65. https://doi.org/10. 1093/infdis/jix410.

12. Pinti M, Appay V, Campisi J, et al. Aging of the immune system: Focus on inflammation and vaccination. Eur J Immunol. 2016;46(10):2286-301. https://doi.org/10.1002/eji.201546178.

13. Butcher S, Chahel H, Lord JM. Review article: ageing and the neutrophil: no appetite for killing? Immunology. 2000;100(4):411-6.

14. Butcher SK, Chahal H, Nayak L, et al. Senescence in innate immune responses: reduced neutrophil phagocytic capacity and CD16 expression in elderly humans. J Leukoc Biol. 2001;70(6):881-6.

15. Romero-Steiner S, Musher DM, Cetron MS, et al. Reduction in functional antibody activity against Streptococcus pneumoniae in vaccinated elderly individuals highly correlates with decreased IgG antibody avidity. Clin Infect Dis. 1999;29(2):281-8. https:// doi.org/10.1086/520200.

16. Bahler C, Huber CA, Brungger B, Reich O. Multimorbidity, health care utilization and costs in an elderly community-dwelling population: a claims data based observational study. BMC Health Serv Res. 2015;15:23. https://doi.org/10.1186/s12913-015-0698-2.

17. Hoeck S, Francois G, Geerts J, Van der Heyden J, Vandewoude M, Van Hal G. Health-care and home-care utilization among frail elderly persons in Belgium. Eur J Public Health. 2012;22(5):6717. https://doi.org/10.1093/eurpub/ckr133.

18. Kawai K, Gebremeskel BG, Acosta CJ. Systematic review of incidence and complications of herpes zoster: towards a global perspective. BMJ Open. 2014;4(6):e004833. https://doi.org/10. 1136/bmjopen-2014-004833. 
19. Rajagopalan S. Tuberculosis and aging: a global health problem. Clin Infect Dis. 2001;33(7):1034-9. https://doi.org/10.1086/ 322671.

20. Ademokun A, Wu YC, Martin V, et al. Vaccination-induced changes in human B-cell repertoire and pneumococcal IgM and IgA antibody at different ages. Aging Cell. 2011;10(6):922-30. https://doi.org/10.1111/j.1474-9726.2011.00732.x.

21. Schenkein JG, Park S, Nahm MH. Pneumococcal vaccination in older adults induces antibodies with low opsonic capacity and reduced antibody potency. Vaccine. 2008;26(43):5521-6. https:// doi.org/10.1016/j.vaccine.2008.07.071.

22. Li S, Sullivan NL, Rouphael N, et al. Metabolic phenotypes of response to vaccination in humans. Cell. 2017;169(5):862-77.e17. https://doi.org/10.1016/j.cell.2017.04.026.

23. Zenin A, Tsepilov Y, Sharapov S, et al. Identification of 12 genetic loci associated with human healthspan. Commun Biol. 2019;2:41. https://doi.org/10.1038/s42003-019-0290-0.

24. Blagosklonny MV. From causes of aging to death from COVID19. Aging (Albany NY). 2020;12(11):10004-21. https://doi.org/ 10.18632/aging.103493.

25. Furman D, Campisi J, Verdin E, et al. Chronic inflammation in the etiology of disease across the life span. Nat Med. 2019;25(12):1822-32. https://doi.org/10.1038/ s41591-019-0675-0.

26. Braga-Neto UM, Marques ET Jr. From functional genomics to functional immunomics: new challenges, old problems, big rewards. PLoS Comput Biol. 2006;2(7):e81. https://doi.org/10. 1371/journal.pcbi.0020081.

27. Sette A, Fleri W, Peters B, Sathiamurthy M, Bui HH, Wilson S. A roadmap for the immunomics of category A-C pathogens. Immunity. 2005;22(2):155-61. https://doi.org/10.1016/j.immuni. 2005.01.009.

28. Levine ME. Modeling the rate of senescence: can estimated biological age predict mortality more accurately than chronological age? J Gerontol A Biol Sci Med Sci. 2013;68(6):667-74. https:// doi.org/10.1093/gerona/gls233.

29. Levine ME, Lu AT, Quach A, et al. An epigenetic biomarker of aging for lifespan and healthspan. Aging. 2018;10(4):573-91. https://doi.org/10.18632/aging.101414.

30. Belsky DW, Caspi A, Houts R, et al. Quantification of biological aging in young adults. Proc Natl Acad Sci U S A. 2015;112(30):E4104-10. https://doi.org/10.1073/pnas.15062 64112.

31. Sanchez-Niubo A, Forero CG, Wu YT, et al. Development of a common scale for measuring healthy ageing across the world: results from the ATHLOS consortium. Int J Epidemiol. 2020. https://doi.org/10.1093/ije/dyaa236.

32. Fuchs J, Scheidt-Nave C, Hinrichs $\mathrm{T}$, et al. Indicators for healthy ageing-a debate. Int $J$ Environ Res Public Health. 2013;10(12):6630-44. https://doi.org/10.3390/ijerph10126630.

33. Sanders JL, Minster RL, Barmada MM, et al. Heritability of and mortality prediction with a longevity phenotype: the healthy aging index. J Gerontol A Biol Sci Med Sci. 2014;69(4):479-85. https:// doi.org/10.1093/gerona/glt117.

34. López-Otín C, Blasco MA, Partridge L, Serrano M, Kroemer G. The hallmarks of aging. Cell. 2013;153(6):1194-217. https://doi. org/10.1016/j.cell.2013.05.039.

35. Pusceddu I, Kleber M, Delgado G, Herrmann W, März W, Herrmann M. Telomere length and mortality in the Ludwigshafen Risk and Cardiovascular Health study. PLoS ONE. 2018;13(6):e0198373. https://doi.org/10.1371/journal.pone.01983 73.

36. Deelen J, Kettunen J, Fischer K, et al. A metabolic profile of allcause mortality risk identified in an observational study of 44,168 individuals. Nat Commun. 2019;10(1):3346. https://doi.org/10. 1038/s41467-019-11311-9.
37. Liu Z, Kuo PL, Horvath S, Crimmins E, Ferrucci L, Levine M. A new aging measure captures morbidity and mortality risk across diverse subpopulations from NHANES IV: A cohort study. PLoS Med. 2018;15(12):e1002718. https://doi.org/10.1371/journal. pmed.1002718.

38. Kuo CL, Pilling LC, Atkins JC, et al. COVID-19 severity is predicted by earlier evidence of accelerated aging. medRxiv. 2020. doi:https://doi.org/10.1101/2020.07.10.20147777

39. Ahadi S, Zhou W, Schüssler-Fiorenza Rose SM, et al. Personal aging markers and ageotypes revealed by deep longitudinal profiling. Nat Med. 2020;26(1):83-90. https://doi.org/10.1038/ s41591-019-0719-5.

40. Crooke SN, Ovsyannikova IG, Poland GA, Kennedy RB. Immunosenescence: A systems-level overview of immune cell biology and strategies for improving vaccine responses. Exp Gerontol. 2019;124:110632. https://doi.org/10.1016/j.exger.2019.110632.

41. Aiello A, Farzaneh F, Candore G, et al. Immunosenescence and its hallmarks: how to oppose aging strategically? A review of potential options for therapeutic intervention. Front Immunol. 2019;10:2247.

42. Kuningas M, May L, Tamm R, et al. Selection for genetic variation inducing pro-inflammatory responses under adverse environmental conditions in a Ghanaian population. PLoS ONE. 2009;4(11):e7795. https://doi.org/10.1371/journal.pone.0007795.

43. Dhillon BK, Smith M, Baghela A, Lee AHY, Hancock REW. Systems Biology Approaches to Understanding the Human Immune System. Front Immunol. 2020;11:1683. https://doi.org/10.3389/ fimmu.2020.01683.

44. Pulendran B, Davis MM. The science and medicine of human immunology. Science. 2020;369(6511). doi:https://doi.org/10. 1126/science.aay4014

45. Lee AH, Shannon CP, Amenyogbe N, et al. Dynamic molecular changes during the first week of human life follow a robust developmental trajectory. Nat Commun. 2019;10(1):1092. https://doi. org/10.1038/s41467-019-08794-x.

46. Alpert A, Pickman Y, Leipold $M$, et al. A clinically meaningful metric of immune age derived from high-dimensional longitudinal monitoring. Nat Med. 2019;25(3):487-95. https://doi.org/10. 1038/s41591-019-0381-y.

47. Lu LL, Chung AW, Rosebrock TR, et al. A functional role for antibodies in tuberculosis. Cell. 2016;167(2):433-43.e14. https:// doi.org/10.1016/j.cell.2016.08.072.

48. Jennewein MF, Goldfarb I, Dolatshahi S, et al. Fc glycan-mediated regulation of placental antibody transfer. Cell. 2019;178(1):20215.e14. https://doi.org/10.1016/j.cell.2019.05.044.

49. Franceschi C, Campisi J. Chronic inflammation inflammaging and its potential contribution to age-associated diseases. J Gerontol A Biol Sci Med Sci. 2014;69(Suppl 1):S4-9. https://doi.org/10. 1093/gerona/glu057.

50. Gibson KL, Wu YC, Barnett Y, et al. B-cell diversity decreases in old age and is correlated with poor health status. Aging Cell. 2009;8(1):18-25. https://doi.org/10.1111/j.1474-9726.2008. 00443.x.

51. Yanes RE, Gustafson CE, Weyand CM, Goronzy JJ. Lymphocyte generation and population homeostasis throughout life. Semin Hematol. 2017;54(1):33-8. https://doi.org/10.1053/j.seminhemat ol.2016.10.003.

52. Furman D, Chang J, Lartigue L, et al. Expression of specific inflammasome gene modules stratifies older individuals into two extreme clinical and immunological states. Nat Med. 2017;23(2):174-84. https://doi.org/10.1038/nm.4267.

53. Gritsenko A, Green JP, Brough D, Lopez-Castejon G. Mechanisms of NLRP3 priming in inflammaging and age related diseases. Cytokine Growth Factor Rev. 2020. https://doi.org/10. 1016/j.cytogfr.2020.08.003. 
54. Schroder K, Tschopp J. The inflammasomes. Cell. 2010;140(6):821-32. https://doi.org/10.1016/j.cell.2010.01.040.

55. Hirako IC, Gallego-Marin C, Ataide MA, et al. DNA-containing immunocomplexes promote inflammasome assembly and release of pyrogenic cytokines by CD14+ CD16+ CD64high CD32low inflammatory monocytes from malaria patients. mBio. 2015;6(6):e0160515. https://doi.org/10.1128/mBio.01605-15.

56. Müller-Calleja N, Köhler A, Siebald B, et al. Cofactor-independent antiphospholipid antibodies activate the NLRP3-inflammasome via endosomal NADPH-oxidase: implications for the antiphospholipid syndrome. Thromb Haemost. 2015;113(5):1071-83. https://doi.org/ 10.1160/th14-07-0628.

57. Ruhaak LR, Uh HW, Beekman M, et al. Decreased levels of bisecting GlcNAc glycoforms of $\mathrm{IgG}$ are associated with human longevity. PLoS ONE. 2010;5(9):e12566. https://doi.org/10.1371/journal.pone. 0012566.

58. Gudelj I, Lauc G, Pezer M. Immunoglobulin G glycosylation in aging and diseases. Cell Immunol. 2018;333:65-79. https://doi.org/ 10.1016/j.cellimm.2018.07.009.

59. Irvine EB, Alter G. Understanding the role of antibody glycosylation through the lens of severe viral and bacterial diseases. Glycobiology. 2020;30(4):241-53. https://doi.org/10.1093/glycob/cwaa018.

60. Gunn BM, Alter G. Modulating Antibody Functionality in Infectious Disease and Vaccination. Trends Mol Med. 2016;22(11):969-82. https://doi.org/10.1016/j.molmed.2016.09.002.

61. Kristic J, Vuckovic F, Menni C, et al. Glycans are a novel biomarker of chronological and biological ages. J Gerontol A Biol Sci Med Sci. 2014;69(7):779-89. https://doi.org/10.1093/gerona/glt190.

62. Plomp R, Ruhaak LR, Uh HW, et al. Subclass-specific IgG glycosylation is associated with markers of inflammation and metabolic health. Sci Rep. 2017;7(1):12325. https://doi.org/10.1038/ s41598-017-12495-0.

63. Sorgdrager FJH, Naudé PJW, Kema IP, Nollen EA, Deyn PP. Tryptophan metabolism in inflammaging: from biomarker to therapeutic target. Front Immunol. 2019;10:2565. https://doi.org/10.3389/fimmu. 2019.02565 .

64. Próchnicki T, Latz E. Inflammasomes on the crossroads of innate immune recognition and metabolic control. Cell Metab. 2017;26(1):71-93. https://doi.org/10.1016/j.cmet.2017.06.018.

65. Cai Y, Kim DJ, Takahashi T, et al. Kynurenic acid underlies sexspecific immune responses to COVID-19. medRxiv. 2020. doi:https:// doi.org/10.1101/2020.09.06.20189159

66. Shen B, Yi X, Sun Y, et al. Proteomic and metabolomic characterization of COVID-19 patient sera. Cell. 2020;182(1):59-72.e15. https:// doi.org/10.1016/j.cell.2020.05.032.

67. Song JW, Lam SM, Fan X, et al. Omics-driven systems interrogation of metabolic dysregulation in COVID-19 pathogenesis. Cell Metab. 2020;32(2):188-202.e5. https://doi.org/10.1016/j.cmet.2020.06.016.

68. Delafiori J, Navarro LC, Siciliano RF, et al. Covid-19 automated diagnosis and risk assessment through metabolomics and machinelearning. Anal Chem. 2021;93(4):2471-9. https://doi.org/10.1021/ acs.analchem.0c04497.

69. Fan J, Wang H, Ye G, et al. Letter to the Editor: Low-density lipoprotein is a potential predictor of poor prognosis in patients with coronavirus disease 2019. Metabolism. 2020;107:154243. https://doi. org/10.1016/j.metabol.2020.154243.

70. Salimi S, Hamlyn JM. COVID-19 and crosstalk with the hallmarks of aging. J Gerontol A Biol Sci Med Sci. 2020;75(9):34-41. https:// doi.org/10.1093/gerona/glaa149.

71. Lara PC, Macías-Verde D, Burgos-Burgos J. Age-induced NLRP3 inflammasome over-activation increases lethality of SARS-CoV-2 pneumonia in elderly patients. Aging Dis. 2020;11(4):756-62. https:// doi.org/10.14336/ad.2020.0601.

72. Larsen MD, de Graaf EL, Sonneveld ME, et al. Afucosylated IgG characterizes enveloped viral responses and corelates with COVID-19 severity. Science. 2021;371(6532):eabc8378. https://doi.org/10.1126/ science.abc 8378

73. Chakraborty S, Edwards K, Buzzanco AS, et al. Symptomatic SARSCoV-2 infections display specific IgG Fc structures. medRxiv. 2020. doi:https://doi.org/10.1101/2020.05.15.20103341

74. Akbar AN, Gilroy DW. Aging immunity may exacerbate COVID19. Science. 2020;369(6501):256-7. https://doi.org/10.1126/science. abb0762.

75. Bermejo-Martin JF, Gonzalez-Rivera M, Almansa R, et al. Viral RNA load in plasma is associated with critical illness and a dysregulated host response in COVID-19. Crit Care. 2020;24(1):691. https:// doi.org/10.1186/s13054-020-03398-0.

76. Zheng Y, Liu X, Le W, et al. A human circulating immune cell landscape in aging and COVID-19. Protein Cell. 2020:1-31. doi:https:// doi.org/10.1007/s13238-020-00762-2

77. Chung AW, Alter G. Systems serology: profiling vaccine induced humoral immunity against HIV. Retrovirology. 2017;14(1):57. https:// doi.org/10.1186/s12977-017-0380-3.

78. Chung AW, Kumar MP, Arnold KB, et al. Dissecting polyclonal vaccine-induced humoral immunity against HIV using systems serology. Cell. 2015;163(4):988-98. https://doi.org/10.1016/j.cell.2015.10.027.

79. Isnard $\mathrm{P}$, Kula $\mathrm{T}$, Avettand Fenoel V, et al. Temporal virus serological profiling of kidney graft recipients using VirScan. Proc Natl Acad Sci U S A. 2019;116(22):10899-904. https://doi.org/10.1073/pnas.18211 66116.

80. Larman HB, Zhao Z, Laserson U, et al. Autoantigen discovery with a synthetic human peptidome. Nat Biotechnol. 2011;29(6):535-41. https://doi.org/10.1038/nbt.1856.

81. Mina MJ, Kula T, Leng Y, et al. Measles virus infection diminishes preexisting antibodies that offer protection from other pathogens. Science. 2019;366(6465):599-606. https://doi.org/10.1126/science. aay6485.

82. Shrock E, Fujimura E, Kula T, et al. Viral epitope profiling of COVID-19 patients reveals cross-reactivity and correlates of severity. Science. 2020;370(6520):eabd4250. https://doi.org/10.1126/scien ce.abd4250.

83. Xu GJ, Kula T, Xu Q, et al. Viral immunology. Comprehensive serological profiling of human populations using a synthetic human virome. Science. 2015;348(6239):aaa0698. https://doi.org/10.1126/ science.aaa0698.

84. Lauc G, Huffman JE, Pučić M, et al. Loci associated with N-glycosylation of human immunoglobulin $\mathrm{G}$ show pleiotropy with autoimmune diseases and haematological cancers. PLoS Genet. 2013;9(1):e1003225. https://doi.org/10.1371/journal.pgen.1003225.

85. Topol E. High-performance medicine: the convergence of human and artificial intelligence. Nature Medicine. 2019;25:44-56. https://doi.org/10.1038/s41591-018-0300-7.

86. Yu K, Beam AL, Kohane IS. Artificial intelligence in healthcare. Nat Biomed Eng. 2018;2(10):719-31. https://doi.org/10.1038/ s41551-018-0305-z.

87. Zou H, Hastie T. Regularization and variable selection via the elastic net. J R Stat Soc. 2005;67:301-20. https://doi.org/10. 1111/j.1467-9868.2005.00503.x.

88. Breiman L. Random forests. Mach Learn. 2001;45:5-32. https:// doi.org/10.1023/A:1010933404324.

89. Hochreiter S, Schmidhuber J. Long short-term memory. Neural Comput. 1997;9:1735-80.

Publisher's Note Springer Nature remains neutral with regard to jurisdictional claims in published maps and institutional affiliations. 